\title{
A ELETRODINÂMICA DE WEBER E SEUS DESENVOLVIMENTOS RECENTES
}

\author{
André Koch Torres Assis 1 \\ Instituto de Física "Gleb Watahin" \\ Unicamp - Campinas, SP
}

\section{RESUMO}

É apresentada a eletrodinâmica desenvolvida por Wilhelr.ı Weber e suas principais propriedades (conservação do momento linear, do momento angular e da energia). Indica-se o fato de se derivar as equações de Maxwell a partir da eletrodinâmica de Weber. Também a força entre elementos de corrente de Ampère pode ser derivada a partir dela. Apresentam-se resultados experimentais relacionados com a eletrodinâmica de Weber tais como: experimentos ligados com a força de Ampère e com a força de Grassmann-Biot-Savart; e a existência ou não de um campo elétrico devido a uma corrente elétrica neutra, constante e estacionária. Por último discute-se desenvolvimentos recentes da eletrodinâmica de Weber: introdução do tempo retardado; a existência de termos de ordem superior a $v^{2} / c^{2}$; e a possibilidade de derivação da gravitação a partir de uma generalização da força de Weber.

Palavras Chave: Eletrodinâmica de Weber, força de Ampere entre elementos de corrente, tempo retardado.

\begin{abstract}
We present the eletrodynamics developed by Wilhelm Weber and its main properties (conservation of linear momentum, angular momentum and energy).

We show how Maxwell's equations can be derived from Weber's eletrodynamics. Ampere's force between current elements can also be derived from it. We discuss experiments related to Ampere's force and to the existence or not of an electric field outside a dc current carrying wire. Finally we avalyse recent theoretical developments in Weber's electrodynamics: introduction of the retarded time and the existence of terms of third and higher orders of $\mathrm{v}^{2} / \mathrm{c}^{2}$ in a generalized Weber's force.
\end{abstract}

\footnotetext{
${ }^{1}$ Também professor colaborador do Departamento de Matemática Aplicada, IMECC, UNICAMP, 13081-970 Campinas, SP
} 


\section{INTRODUÇÃO}

Wilhem Weber (1804-1891) foi um fisico experimental alemão que trabalhou com o matemático Gauss em Gottingen. Para uma biografia de Weber ver (Assis 1991 a).

Neste trabalho descreveremos a força e a energia de Weber, enfatizando suas propriedades e desenvolvimentos recentes. Os trabalhos de Weber podem ser encontrados em suas obras completas, em alemão, sendo que a parte principal do seu trabalho já se encontra traduzida para o inglês: (Weber 1848, 1851, 1871 e 1892 - 1894). As principais referências onde se pode encontrar uma descrição bem ampla da eletrodinâmica de Weber são: (Maxwell 1954), (Whittaker 1973), (O'Rahilly 1965) e (Wesley 1990). Recentemente publicamos um curso sobre a eletrodinâmica de Weber para quem nunca viu o assunto: (Assis 1992 a). Neste livro além de um número muito grande de referências se encontra um tratamento detalhado de muitas coisas de que trataremos aqui. Uma versão grandemente ampliada e melhorada deste livro se encontra em (Assis 1994).

En 1846 Weber apresentou uma lei de força entre cargas elétricas com o objetivo de unificar a eletrostática (lei de Coulomb) com a eletrodinâmica e o magnetismo (força de Ampère entre elementos de corrente). Esta força pode ser escrita como

$$
F=\frac{q_{1} q_{2}}{4 \pi \varepsilon_{0}} \frac{1}{r^{2}}\left(1-\frac{\dot{r}^{2}}{2 c^{2}}+\frac{r \ddot{r}}{c^{2}}\right)
$$

Esta força é sempre ao longo da reta que une as duas cargas $q_{1}$ e $q_{2}$ (isto é, segue a lei de ação e reação na forma forte), é repulsiva quando positiva e atrativa quando negativa. Devido a este fato a força de Weber satisfaz aos principios de conservação do momento linear e angular. A constante $\varepsilon_{o}$ é a permissividade do vácuo $\left(\varepsilon_{o}=8,85 \times 10^{-12} C^{2} N^{-1} m^{-2}\right), r$ é a distância entre as cargas, $\dot{r}$ é a velocidade radial relativa entre elas $(\dot{r}=d r / d t)$ e $\ddot{r}$ é a aceleração radial relativa entre elas $\left(\ddot{r}=d^{2} r / d t^{2}\right)$. A constante $c$ que aparece nesta equação é a razão entre as unidades eletromagnéticas e eletrostáticas de carga. Seu valor foi determinado experimentalmente pela primeira vez por Weber e Kohlrausch, em 1856. Obtiveram o valor $c=3,1 \times 10^{8} \mathrm{~ms}^{-1}$ o que foi uma das primeiras indicações quantitativas precisas de uma relação entre o eletromagnetismo e a óptica, já que este era o mesmo valor conhecido da velocidade da luz no vácuo.

Podemos escrever esta equação em termos vetoriais. Sejam $\vec{r}_{1}=x_{1} \hat{x}+y_{1} \hat{y}+z_{1} \hat{z}$ e $\vec{r}_{2}=x_{2} \hat{x}+y_{2} \hat{y}+z_{2} \hat{z}$ as posições das cargas 1 e 2 em relação a um referencial inercial. Podemos definir $\vec{v}_{i}=d \vec{r}_{i} / d t$ e $\vec{a}_{i}=d^{2} \vec{r}_{i} / d t^{2}$, onde $\mathrm{i}=1,2$. Definimos também 
$\vec{r}_{12}=\vec{r}_{1}-\vec{r}_{2}, \vec{v}_{12}=\vec{v}_{1}-\vec{v}_{2}, \vec{a}_{12}=\vec{a}_{1}-\vec{a}_{2} .0$ vetor unitário apontando de 2 para 1 é definido como: $\hat{r}_{12}=\left(\vec{r}_{1}-\vec{r}_{2}\right) / r$. Com isto vem:

$$
\begin{aligned}
& r=\left|\vec{r}_{1}-\vec{r}_{2}\right|=\sqrt{\left(x_{1}-x_{2}\right)^{2}+\left(y_{1}-y_{2}\right)^{2}+\left(z_{1}-z_{2}\right)^{2}} \\
& \dot{r}=\frac{d r}{d t}=\hat{r}_{12} \cdot \vec{v}_{12} \\
& \ddot{r}=\frac{d^{2} r}{d t^{2}}=\frac{d \dot{r}}{d t}=\frac{1}{r}\left[\left(\vec{v}_{12} \cdot \vec{v}_{12}\right)-\left(\hat{r}_{12} \cdot \vec{v}_{12}\right)^{2}+\vec{r}_{12} \cdot \vec{a}_{12}\right]
\end{aligned}
$$

Aplicando todas estas coisas na equação (1) vem então a força de Weber em linguagem vetorial. Nesta forma a força exercida pela carga 2 na carga 1 é dada por:

$$
\vec{F}=\frac{q_{1} q_{2}}{4 \pi \varepsilon_{0}} \frac{\hat{r}_{12}}{r^{2}}\left[1+\frac{1}{c^{2}}\left(\vec{v}_{12} \cdot \vec{v}_{12}-\frac{3}{2}\left(\hat{r}_{12} \cdot \vec{v}_{12}\right)^{2}+\vec{r}_{12} \cdot \vec{a}_{12}\right)\right]
$$

Este foi o primeiro exemplo que surgiu na fisica de uma força que dependia não apenas da distância entre as cargas mas também de suas velocidades e acelerações. Na época se pensou que esta força não conservava a energia até que em 1871 Weber mostrou que esta força também satisfazia ao princípio de conservação de energia. A energia potencial de Weber é dada por

$$
U=\frac{q_{1} q_{2}}{4 \pi \varepsilon_{0}} \frac{1}{r}\left(1-\frac{\dot{r}^{2}}{2 c^{2}}\right)
$$

Seja a energia cinética das duas cargas dada por

$$
T=m_{1} \frac{\vec{v}_{1} \cdot \vec{v}_{1}}{2}+m_{2} \frac{\vec{v}_{2} \cdot \vec{v}_{2}}{2}
$$

É então fácil mostrar que a energia total $E=T+U$ é uma constante, ou seja, $d E / d t$ $=0$. Isto também pode ser mostrado utilizando a Lagrangiana de Weber que é dada por $L=T-S$, onde $S$ é dado por

$$
S=\frac{q_{1} q_{2}}{4 \pi \varepsilon_{0}} \frac{1}{r}\left(1+\frac{\dot{r}^{2}}{2 c^{2}}\right)
$$


Deve-se observar que a energia Lagrangiana $S$ difere de $U$ por uma mudança de sinal na frente de $\dot{r}^{2}$. O mesmo ocorre no eletromagnetismo clássico usual com a Lagrangiana e Hamiltoniana de Darwin.

Com isto vê-se então que a eletrodinâmica de Weber é compativel com todos os princípios de conservação da fisica clássica, ou seja, do momento linear, angular e da energia.

A força de Weber é uma generalização da força de Coulomb retornando a esta quando não há movimento relativo entre as cargas. Logo todos os resultados da eletrostática assim como a lei de Gauss (primeira das equações de Maxwell) são apenas um caso particular da eletrodinâmica de Weber, já que podem ser derivadas desta.

Não vamos entrar em detalhes aqui, mas também a lei de indução de Faraday pode ser derivada da eletrodinâmica de Weber, como foi mostrado pelo próprio Weber. Uma prova disto pode ser encontrada nos livros de Maxwell, Whittaker e O'Rahilly citados anteriormente, assim como em (Assis 1992 a) e (Assis 1994).

Vamos agora discutir outros aspectos da lei de Weber e seus desenvolvimentos recentes.

\section{FORÇAS ENTRE ELEMENTOS DE CORRENTE DE AMPÈRE E DE GRASSMANN- BIOT-SAVART}

Em seguida aos experimentos de Oersted de 1820 que mostraram uma ligação entre as correntes elétricas e o magnetismo, Ampère realizou uma série de experimentos clássicos muito famosos e a partir deles chegou (1826) naquilo que se conhece como a força de Ampère entre os elementos de corrente $I_{1} d \vec{l}_{1}$ e $I_{2} d l_{2}$. Em linguagem vetorial vem que a força de Ampère exercida pelo elemento 2 no elemento 1 é dada por

$$
d^{2} \vec{F}=-\frac{\mu_{0}}{4 \pi} I_{1} I_{2} \frac{\hat{r}_{12}}{r^{2}}\left[2\left(d \vec{l}_{1} \cdot d \vec{l}_{2}\right)-3\left(\hat{r}_{12} \cdot d \vec{l}_{1}\right)\left(\hat{r}_{12} \cdot d \vec{l}_{2}\right)\right]
$$

Nesta equação temos $\mu_{0}=4 \pi \times 10^{-7} \mathrm{kgm} \mathrm{C}^{-2}$ que é uma constante chamada de permeabilidade do vácuo. Um dos aspectos importantes a observar nesta equação é que a força de Ampère sempre satisfaz ao princípio de ação e reação na forma forte, ou seja, a força entre os dois elementos de corrente está sempre ao longo da reta que une os dois elementos, independente de suas orientações. 
De acordo com Maxwell esta expressão de força "deveria sempre permanecer como a mais importante da eletrodinâmica" (Maxwell 1954, Volume 2, Artigo 528, página 175). Infelizmente parece que os fisicos posteriores a Maxwell se esquecerem desta sugestão de Maxwell e hoje em dia não mais se encontra a equação (9) em quase nenhum livro didático que trate do eletromagnetismo (Feynman, Jackson, Panofsky e Phillips, Halliday e Resnick, Purcell, Reitz e Milford, Sears, Tipler, etc.) Em vez da força de Ampère a única expressão de força que aparece é a proposta pelo matemático Grassmann em 1845 dada por:

$$
\begin{aligned}
& d^{2} \vec{F}=I_{1} d \vec{l}_{1} \times d \vec{B}_{2}=I_{1} d \vec{l}_{1} \times\left[\frac{\mu_{0}}{4 \pi} \frac{1}{r^{2}}\left(I_{2} d \vec{l}_{2} \times \hat{r}_{12}\right)\right] \\
& =-\frac{\mu_{0}}{4 \pi} \frac{I_{1} I_{2}}{r^{2}}\left[\left(d \vec{l}_{1} \cdot d \vec{l}_{2}\right) \hat{r}_{12}-\left(d \vec{l}_{1} \cdot \hat{r}_{12}\right) d \vec{l}_{2}\right]
\end{aligned}
$$

Esta lei de força é baseada no campo magnético de Biot-Savart (1820)

Alguns aspectos devem ser enfatizados aqui. Em primeiro lugar deve-se observar que a força de Grassmann não satisfaz em geral ao princípio de ação e reação, a não ser em alguns casos particulares. Por outro lado quando se calcula a força de um circuito fechado de forma arbitrária num elemento de corrente de um outro circuito as forças de Ampère e de Grassmann coincidem. Com isto elas não podem ser distinguidas quando se fazem experimentos envolvendo dois ou mais circuitos fechados. Este é um dos principais motivos pelos quais por muito tempo se pensou que as duas expressões de força fossem equivalentes. Apenas nos últimos 10 anos começaram a ser realizados experimentos com circuito único tentando distinguir as duas expressões. A idéia básica é tentar medir as forças e tensões em parte de um circuito devido ao restante dele. Esta parte do circuito pode estar ligada ao restante por mercúrio liquido, que é condutor de eletricidade, ou por um arco elétrico. Não vamos entrar nos detalhes destes experimentos mas citamos aqui um artigo de revisão e um livro tratando exclusivamente deste assunto: (Graneau 1985 e 1986). Embora muitos destes experimentos favoreçam à força de Ampère em comparação com a de Grassmann, ainda é cedo para se dizer que a questão está definida.

A relação de tudo isto com a eletrodinâmica de Weber é que Weber chegou em sua expressão de força, equação (1), conhecendo a força de Ampère e criando sua equação de tal maneira que ela fosse compativel com a eletrostática (força de Coulomb) e com a eletrodinâmica (força de Ampère). Pode-se fazer também o caminho inverso, ou seja, começar com a força de Weber e se derivar daí as forças de Coulomb (isto é imediato) e de Ampère. Neste último caso 
assume-se que em cada elemento de corrente há um número igual de portadores de carga positiva e negativa, sendo então neutros eletricamente, e soma-se a força de Weber entre cada par de cargas (força das positivas num elemento nas positivas e negativas do outro elemento, e força das negativas num elemento nas positivas e negativas do outro). Quando se faz a soma destes quatro termos e se faz a substituição $d q_{i}\left(\vec{v}_{i+}-\vec{v}_{i-}\right) \rightarrow I_{i} d \vec{l}_{i}$, onde $\mathrm{i}=1,2$, se reobtem a força de Ampère.

Vê-se então que esta questão da controvérsia experimental entre as forças de Ampère e de Grassmann é uma das questões mais importantes relacionadas com a eletrodinâmica de Weber nos últimos anos. Vale a pena enfatizar que Maxwell derivou a lei circuital de Ampère e a lei da nulidade da divergência do campo magnético (duas das equações de Maxwell) a partir da força de Ampère entre elementos de corrente. Deve-se lembrar também que Maxwell conhecia não apenas a força de Ampère mas também a de Grassmann. E em seu livro mais importante, o Treatise, Maxwell faz uma comparação entre estas duas expressões de força e duas outras que ele havia inventado (e que também se reduzem à expressão de Ampère no caso da interação entre dois ou mais circuitos fechados) e faz a seguinte afirmação: "A força de Ampère é sem dúvida a melhor, pois ela é a única que faz as forças sobre os dois elementos não apenas iguais e opostas mas ao longo da linha reta que os une" (Maxwell 1954, Volume 2, Artigo 527, página 174).

\section{DESENVOLVIMENTOS RECENTES DA ELETRODINÂMICA DE WEBER}

Agora vamos tratar de outros aspectos. Recentemente aplicamos uma força de Weber para analisar os famosos experimentos de Kaufmann e Bucherer (Assis 1989 a), (Assis e Caluzi 1991). Estes experimentos são sempre citados como provas experimentais da variação da massa com a velocidade. Nestes experimentos uma fonte de elétrons era colocada no meio de um capacitor largo que gerava um campo elétrico uniforme, $E=\sigma / \varepsilon_{0}$, onde $\sigma$ é a densidade superficial de carga nas placas do capacitor. Em direção normal ao movimento dos elétrons e ao campo elétrico era colocado um campo magnético uniforme. Estes dois campos cruzados funcionavam como um filtro de velocidades de tal forma que os elétrons que conseguiam sair do capacitor (e portanto os que não colidiam com as placas) satisfaziam à condição de que suas velocidades eram dadas por $v=E / B$, onde $B$ é a intensidade do campo magnético. Após sairem do capacitor os elétrons moviam-se apenas numa região de campo magnético uniforme e então descreviam um raio de Larmor. Acontece que o raio observado não coincidia com o que se esperava classicamente, isto é, com $r=m_{0} v / q B$, sendo $v=E / B, m_{0}$ sendo a massa do elétron e $q$ sua carga. A interpretação clássica deste experimento é de que ele mostra que a massa não é uma constante mas que varia com 
a velocidade de acordo com a expressão relativistica $m=m_{0} / \sqrt{1-v^{2} / c^{2}}$. Com $m$ em vez de $m_{0}$ na expressão do raio de Larmor a previsão teórica passa a concordar com o obtido experimentalmente. Como mostramos nos artigos citados, uma outra interpretação é a de que a massa do elétron é uma constante qualquer que seja sua velocidade, mas a expressão de força a ser utilizada deve ser a de Weber e não a de Coulomb simples. Com isto a força dentro do capacitor passa a ser $q \sigma\left(1+v^{2} / 2 c^{2}\right) / \varepsilon_{0}$. Esta interpretação também explicaria os dados experimentais, embora seja bem distinta conceitualmente das explicações usuais.

A força de Weber envolve termos até segunda ordem em v/c. Recentemente Phipps propôs uma generalização da lei de Weber e com isto conseguiu superar as críticas que Helmholtz havia feito à eletrodinâmica de Weber (Phipps 1990 a, b). O potencial de Phipps pode ser expresso como

$$
U_{P}=\frac{q_{1} q_{2}}{4 \pi \varepsilon_{0}} \frac{1}{r} \sqrt{1-\frac{\dot{r}^{2}}{c^{2}}}=\frac{q_{1} q_{2}}{4 \pi \varepsilon_{0}} \frac{1}{r}\left(1-\frac{\dot{r}^{2}}{2 c^{2}}-\frac{\dot{r}^{4}}{8 c^{4}}-\ldots\right)
$$

De acordo com esta idéia a lei de Weber seria apenas uma aproximação válida apenas até segunda ordem em $v / c$ (Assis e Caluzi 1991). Phipps chega a propor que uma generalização mais ampla envolveria também outras derivadas além de $\$$ e embora não chegue a explorar esta idéia.

Já Wesley generaliza a eletrodinâmica de Weber por um outro caminho (Wesley 1990). Uma das características da eletrodinâmica de Weber é o fato de ser uma teoria baseada na ação à distância. Para muitos isto é uma limitação da teoria e para superar este fato Wesley introduziu recentemente o tempo retardado na lei de Weber, isto é, $(t-r / c) \rightarrow t$. Conseguiu assim obter as equações de onda para o potencial escalar elétrico e para o potencial vetor magnético a partir desta generalização de Weber.

No lado experimental além da controvérsia Ampère versus Grassmann há também a dúvida se existe ou não um campo elétrico devido a uma corrente elétrica neutra, estacionária $\mathrm{e}$ constante. Este campo não é previsto pela teoria eletromagnética usual com os potencias de Lienard-Wiechert, mas deveria existir de acordo com as teorias de Weber, Riemann e alguns outros. Há um experimento de 1976 indicando a existência deste campo, como previsto por Weber, um outro experimento de 1977 que não encontra este campo, e um experimento de 1985 que encontra um campo mas apontando na direção contrária à prevista por Weber. Recentemente discutimos todos estes experimentos e os comparamos com a eletrodinâmica de Weber (Assis 1991 b) e portanto não entraremos em detalhes aqui. $\mathrm{O}$ ponto mais importante a ser mencionado é que este ainda é um assunto controverso mas de grande relevância atualmente. 
Um outro desenvolvimento recente é que uma generalização da eletrodinâmica de Weber do tipo da de Phipps permite com que se derive uma força gravitacional similar à de Newton a partir apenas do eletromagnetismo. A idéia é calcular a força média entre dipolos neutros eletricamente nos quais as cargas negativas oscilam em torno das positivas. Fazendo-se todos os cálculos com esta força de Weber generalizada obtem-se uma força média atrativa entre os dipolos que simularia aquilo que chamamos de gravitação já que seria sempre atrativa, cai com o inverso do quadrado da distância, é ao longo da reta que une os dipolos, satisfaz ao principio de ação e reação, e é da ordem de grandeza correta, ou seja, $10^{40}$ vezes menor do que a força eletrostática usual. Para um detalhe de todos estes cálculos e uma discussão histórica e crítica do assunto ver (Assis 1992 b).

Também relacionado com estas idéias de unificação das forças da natureza vale a pena citar um artigo importante onde se mostra uma grande similaridade entre a força de Weber eletromagnética e as forças que descrevem as interações nucleares (Pearson e Kilambi 1974). Esta linha de trabalho parece ser promissora.

Um outro aspecto bem moderno e recente é um estudo da estabilidade das órbitas planetárias utilizando uma força de Weber gravitacional realizado por Solkol'skii e Sadovnikov (1987). Eles utilizaram, em particular, teorias de caos nesta análise.

Um último aspecto que gostariamos de ressaltar é que uma lei de força gravitacional Weberiana, ou seja, uma força tipo Newtoniana mas com termos que dependem da velocidade e aceleração entre as massas permite com que se implemente quantitativamente o princípio de Mach: (Assis 1989 b) e (Assis 1994), Capítulo 7. Este principio diz que a origem da inércia de todos os corpos vem de uma interação gravitacional de qualquer corpo com o restante do universo. Por inércia aqui se entende tanto a massa inercial dos corpos, como qualquer grandeza que envolva esta massa, como a energia cinética e as forças inerciais ( $m \stackrel{r}{a}$, força de Coriolis e força centrífuga). Para Mach as forças "fictícias" são tão reais quanto qualquer outra e têm origem numa interação gravitacional de qualquer corpo com o universo em rotação ou acelerado. Para uma discussão das idéias de Mach sugerimos fortemente seu próprio livro (Mach 1960) e o de Barbour (1989).

Com isto encerramos este artigo. A idéia principal foi de apresentar os aspectos principais da eletrodinâmica de Weber e os desenvolvimentos teóricos e experimentais recentes relacionados a ela.

AGRADECIMENTOS: O autor deseja agradecer a FAPESP, ao CNPq e a FAEP pelo apoio recebido nos últimos anos. 


\section{REFERÊNCIAS}

Assis, A. K. T. (1989 a) Physics Letters A, Volume 136, páginas 277 a 280, "Weber's law and mass variation."

Assis, A. K. T. (1989 b) Foundations of Physics Letters, Volume 2, páginas 301 a 318, "On Mach's principle."

Assis, A. K. T. (1991 a) Revista da Sociedade Brasileira de História da Ciência, Volume 5, páginas 53 a 59, "Wilhelm Eduard Weber (1804 - 1891), sua vida e sua obra."

Assis, A. K. T. (1991 b) Physics Essays, Volume 4, páginas 109 a 114, "Can a steady current generate an electric field?"

Assis, A. K. T. (1992 a) Curso de Eletrodinâmica de Weber (Notas de Física IFGW Número 5, Setor de Publicações do Instituto de Física da UNICAMP, Campinas).

Assis, A. K. T. (1992 b) Canadian Journal of Physics, Volume 70, páginas 330 a 340, "Deriving gravitation from electromagnetism."

Assis, A. K. T. (1994) Weber's Electrodynamics (Kluwer Academic Publishers, Dordrecht)

Assis, A. K. T. e Caluzi, J. J. (1991), Physics Letters A, Volume 160, páginas 25 a 30, "A limitation of Weber's law."

Barbour, J. B. (1989) Absolute or Relative Motion? - A study from a Machian point of view of the discovery and the structure of dynamical theories (Cambridge University Press, Cambridge)

Graneau, P. (1985) Ampere-Neumanm Electrodynamics of Metals (Hadronic Press, Nonantum).

Graneau, P. (1986) Fortschritte der Physik, Volume 34, páginas 457 a 503, "The Ampere-Neumann electrodynamics of metallic conductors."

Mach, E. (1960) The Science of Mechanics - A Critical and Historical Account of its Development (Open Court, La Salle).

Maxwell, J. C. (1954) A Treatise on Electricity and Magnetism, (Dover, New York), Volume 2, Capitulo 23.

O'Rahilly, A. (1965) Electromagnetic Theory - A Critical Examination of Fundamentals (Dover, New York), Volume 2, Capitulo 11.

Pearson, J. M. e Kilambi, A. (1974) American Journal of Physics, Volume 42, páginas 971 a 975, "Velocity-dependent nuclear forces and Weber's electrodynamics."

Phipps Jr., T. E. (1990 a) Physics Essays, Volume 3, páginas 414 a 420, "Toward modernization of Weber's force law."

Phipps Jr., T. E. (1990 b) Apeiron, Volume 8, páginas 8 a 14, "Weber-type laws of action-at-adistance in modern physics."

Solkol'skii, A. G. e Sadovnikov, A. A. (1987) Soviet Astronomy (Astronomical Journal), Volume 31, páginas 90 a 93, "Lagrangian solutions for Weber's law of attraction."

Weber, W. (1848) Annalen der Physik, Volume 73, página 193; tradução para o inglês em R. Taylor (editor), Scientific Memoirs, Volume 5, página 489 a 529 (Johnson's Reprint Corporation, Londres, 1966), "On the measurement of electro-dynamic forces." 
Weber, W. (1851) Annalen der Physik, Volume 82, página 337; tradução para o inglês em Philosophical Magazine, Volume 22, páginas 226 a 240 e 261 a 269 (1851), "On the measurement of electric resistance according to an absolute standard."

Weber, W. (1871) Abhandlungen der mathem.-phys. Classe der Konigl. Sachsischen Gessellschaft der Wissenschaften, Volume 10; tradução para o inglês em Philosophical Magazine, Volume 43, páginas 1 a 20 e 119 a 149 (1872), "Electrodynamic measurements relating specially to the principle of the conservation of energy."

Weber, W. (1892 - 1894) As obras completas de W. Weber podem ser encontradas nas Werke de Wilhelm Weber (Springer, Berlin): Volume 1, Akustik, Mechanik, Optik und Warmelehre, W. Voigt (editor), (1892). Volume 2, Magnetismus, E. Riecke (editor), (1892). Volume 3, Galvanismus und Elektrodynamik, primeira parte, H. Weber (editor), (1893). Volume 4, Galvanismus und Elektrodynamik, segunda parte, H. Weber (editor), (1894). Volume 5, com E. H. Weber, Wellentehre auf Experimente gegrundet oder uber die Wellen tropfbarer Flussigkeiten mit Anwendung auf die Schall-und Lichtwellen, E. Riecke (editor), (1893). Volume 6, Mechanik der Menschlichen Gehwerkzeuge, F. Merkel e O. Fischer (editores), (1894).

Wesley, J. P. (1990) Foundations of Physics Letters, Volume 3, páginas 443 a 469, "Weber electrodynamics, Part I: General theory, steady current effects;" ibidem, páginas 471 a 490, "Part II: Unipolar induction, Z-antenna;" ibidem, páginas 581 a 605, "Part III: Mechanics, gravitation."

Whittaker, E. T.(1973) A History of the Theories of Aether and Electricity (Humanities Press, New York), Volume 1, Capitulo 7. 